\title{
CLEAN PRESSURE DROP OF NON-NEWTONIAN LIQUID FLOW ACROSS STACKED-DISC FILTERS
}

\author{
J. J. MEERMAN and H. J. H. BROUWERS' \\ Akzo Research Laboratories Arnhem, Fibers Division, Department of Mechanical Engineering, \\ Velperweg 76, 6824 BM Arnhem, The Netherlands
}

(Received 28 November 1991; accepted for publication in revised form 5 April 1993)

\begin{abstract}
A bstract-The present paper deals with clean pressure drop and power-law liquid flow across stacked-disc filters. Firstly, from mass and momentum (Darcy's law) balances the governing equations of the process are derived. Subsequently, these equations are reduced to a single dimensionless differential equation whose solution is governed by three dimensionless groups. Numerical and analytical solutions of the equation reveal the effect of these dimerisionless groups on flow and related pressure drop. Finally, the theoretical model of the filter is found to be in good agreement with the performed experiments.
\end{abstract}

\section{INTRODUCTION}

For many years now stacked-disc filters have been widely used in industry for the filtration of liquid polymers, suspensions, and so on. The stacked-disc filters contain a static nest of concentric discs that are separated by filter media, fully incorporated within a pressure-resistant casing (see Fig. 1). The major advantage of the stacked-disc filters is that they contain a great filtration area in combination with a compact construction.

An important property of the filters is the initial pressure drop across the filters, since the maximum pressure in the filter is limited by the mechanical strength of the construction. Moreover, during the operation of the filter the pressure rises due to the pollution of the filter. As soon as the maximum pressure drop is attained, the filtration ends and the filter is washed or dewatered (see Fig. 2). If the filter is dimensioned such that the initial pressure is as low as possible, the actual service time can be extended substantially. Hence, it is obvious that a low initial pressure drop is of utmost importance.

The clean liquid flow and the related pressure drop depend on the geometrical properties of the stackeddisc filter and on the physical properties of the filtered fluid. Booy (1982) theoretically analyzed the initial flow of Newtonian liquids through stacked-disc filters. The initial liquid flow across these filters has been analyzed by Elghossain (1990) to determine the residence time distribution in the filter. The clean pressure drop of non-Newtonian fluids across stackeddisc filters has to the authors' knowledge never been investigated theoretically and experimentally. Accordingly, this analysis is presented here.

To this end, the conservation laws of mass and momentum are employed to describe the power-law (also referred to as Ostwald-de Waele) liquid flow in

'Present address: University of Twente, Department of Civil Engineering and Management, P.O. Box 217, 7500 AE Enschede, The Netherlands. Author to whom correspondence should be addressed. supply gaps, filter medium and discharge gaps. As the Reynolds number of the flow is much smaller than unity, the momentum equation is reduced to the socalled Darcy's law, named after Darcy (1856). The

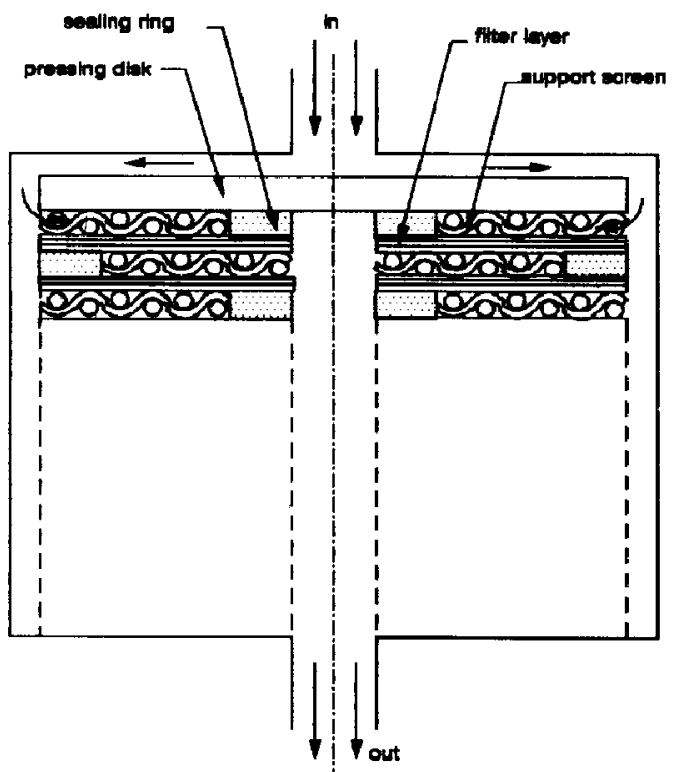

Fig. 1. The stacked-disc filter.

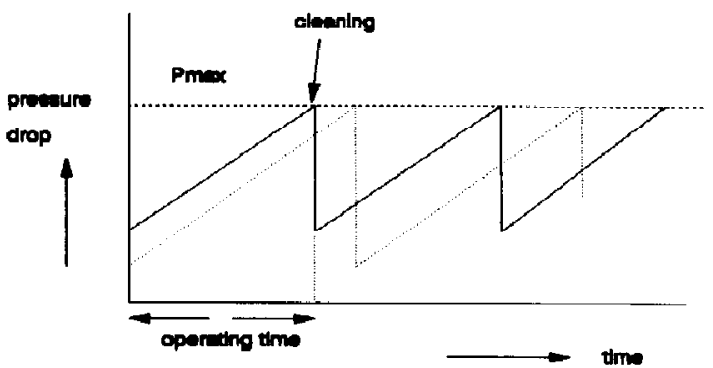

Fig. 2. Pressure drop across a filter as a function of time. 
governing equations are reduced to a single ordinary second-order differential equation and solved. It is demonstrated that the clean pressure drop depends on three dimensionless numbers which contain the properties of construction and fluid. With these numbers expressions are derived which are well suited for the scalc-up of stacked-disc filter constructions. Ultimately, the theoretical predictions are compared with the experimental results, concerning non-Newtonian liquid flow and pressure drop measurements for a broad range of values of the governing dimensionless groups.

\section{FORMULATION OF THE BASIC EQUATIONS}

The stacked-disc filter considered in our present study is constructed from axisymmetric filter media separated by support rings (see Fig. 3). The liquid enters the supply gaps between two inner rings and flows in the $r$-direction. It is assumed that the liquid spreads equally over the various gaps, so that the analysis can be limited to the consideration of one gap. Flowing through a supply gap, part of the liquid passes through the filter medium and continues flowing in the r-direction through the discharge gap. At $r=r_{u}$ all of the liquid has been forced through the filter medium and next the liquid leaves the discharge gap between the outer support rings. The non-Newtonian liquid behavior is represented by the so-called power-law model, discussed among others by Bird et al. (1977).

An overall mass balance of liquid flowing through the supply gap and filter media yields

$$
h \frac{\mathrm{d} u_{1} r}{\mathrm{~d} r}+2 v r=0
$$

and for the discharge gap the balance reads

$$
h \frac{\mathrm{d} u_{z} r}{\mathrm{~d} r}-2 v r=0 .
$$

The factor of 2 in eqs (1) and (2) reckons with the presence of a filter medium on both sides of the gap.

The steady, incompressible and isothermal powerlaw liquid flow across the filter medium can be properly described by Darcy's law:

$$
p_{1}(r)-p_{2}(r)=\frac{t_{f} m_{\text {app. } f} v^{n}}{k_{f}} .
$$

The portrait of the porous filter medium as a bundle of tubes with a hydraulic diameter distribution provides the apparent viscosity $m_{\mathrm{app}, f}$ and the permeability $k_{f}$. Both for random fiber beds and packed spherical beds, Kaplan et al. (1979) and Kozicki and Tiu (1988), respectively, derived expressions for both properties as a function of some microstructure variables. In deriving eq. (3) it has been implicitly assumed that the How in the filter medium in the $r$-direction is negligible, since $\mathrm{d} p / \mathrm{d} z \gg \mathrm{d} p / \mathrm{d} r$ in the medium.

To both gaps Darcy's law is applicable as well:

$$
\frac{\mathrm{d} p_{1}}{\mathrm{~d} r}=-\frac{m_{\mathrm{app}, g} u_{\mathrm{1}}^{n}}{k_{g}}
$$

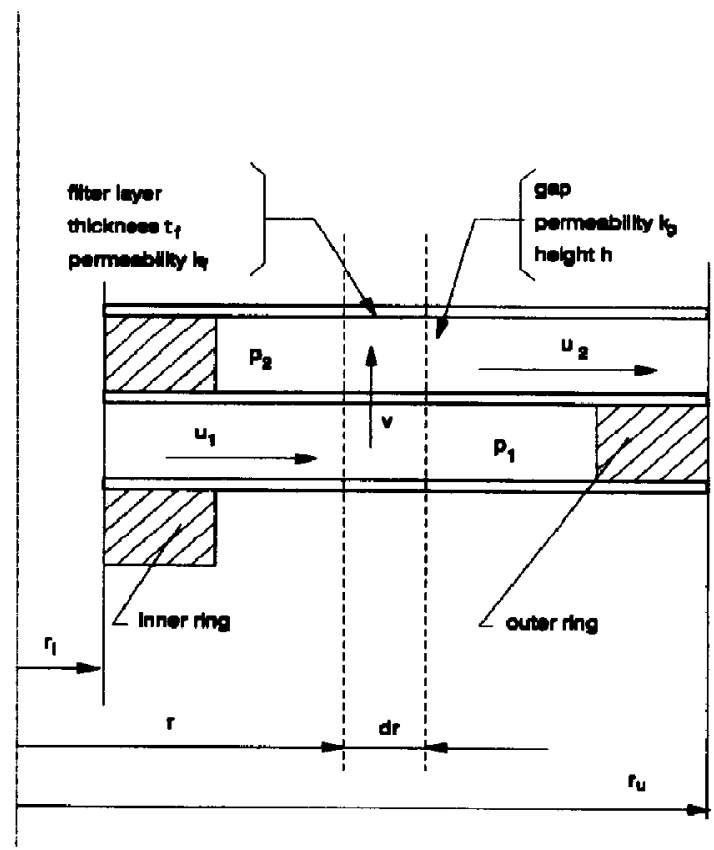

Fig. 3. Schematic liquid flow in the stacked-disc filter.

$$
\frac{\mathrm{d} p_{2}}{\mathrm{~d} r}=-\frac{m_{\mathrm{epp}, g} u_{2}^{n}}{k_{\mathrm{g}}}
$$

since the Reynolds number $R e_{\theta}$ is much smaller than unity. In eqs (4) and (5) $n$ represents the flow behavior index of the power-law fluid. The effect of fluid deceleration and acceleration in the supply and discharge gaps, respectively, can safely be neglected because $v h \rho / 2 \eta \ll 1$; see Berman (1953). Furthermore, as we are interested only in an optimum design of the filter medium and of its supply as well as discharge gaps, we do not consider the pressure drop across the inner and outer support rings.

For an open gap, or slit, the apparent viscosity of the liquid follows from Bird et al. (1977):

$$
m_{\text {app. } \theta}=\frac{m}{6} \frac{(2 / n+4)^{n}}{h^{n-1}}
$$

where $m$ represents the consistency index of the liquid. The permeability of the gap then reads

$$
k_{g}=\frac{1}{12} h^{2} \text {. }
$$

Note that for a Newtonian fiuid the apparent viscosities $m_{\mathrm{app}, \mathrm{g}}$ and $\boldsymbol{m}_{\mathrm{app} .} \boldsymbol{f}$ are both identical to the consistency index $m$, which then corresponds to the Newtonian viscosity. The boundary conditions of eqs (1), (2), (4) and (5) read

$$
\begin{aligned}
& u_{1}\left(r=r_{i}\right)=\frac{\phi}{2 \pi r_{i} h} \\
& u_{2}\left(r=r_{w}\right)=\frac{\phi}{2 \pi r_{w} h}
\end{aligned}
$$




$$
p_{1}\left(r=r_{i}\right)=p_{i} \quad \text { (10) ing }
$$

and

$$
p_{2}\left(r=r_{u}\right)=p_{u}
$$

respectively. In eqs (8) and (9) $\phi$ represents the clean liquid flow that enters and leaves the gaps, while $p_{i}$ and $p_{u}$ are the absolute pressures at the entrance and exit, respectively, of both gaps.

\section{SOLUTHON OF THE GOVERNING EQUATIONS}

In the previous section the governing equations of the fluid flow in the filter have been derived. In this section these equations are reduced to an ordinary non-linear second-order differential equation. This equation is rendered dimensionless and then solved. The solution of the resulting equation provides the pressure drop in the filter as a function of three dimensionless numbers.

Equations (1) and (2) are combined as

$$
v=\frac{h}{4 r} \frac{\mathrm{d}\left(u_{2} r-u_{1} r\right)}{\mathrm{d} r}
$$

and, subsequently, the result is substituted into eq. (3):

$$
p_{1}-p_{2}=\frac{t_{f} m_{\mathrm{app}, f}}{k_{f}}\left[\frac{h}{4 r} \frac{\mathrm{d}\left(u_{2} r-u_{1} r\right)}{\mathrm{d} r}\right]^{n} .
$$

This equation is differentiated with respect to $r$ and eqs (4) and (5) substituted:

$$
\begin{aligned}
-\frac{m_{\mathrm{app}, g}}{k_{\mathrm{s}}}\left(u_{1}^{n}-u_{2}^{n}\right)= & \frac{t_{f} m_{\mathrm{app}, \delta} n}{k_{f}}\left[\frac{h}{4 r} \frac{\mathrm{d}\left(u_{2} r-u_{1} r\right)}{\mathrm{d} r}\right]^{n} \\
& \times \frac{\mathrm{d} \ln \left[\frac{1}{r} \frac{\mathrm{d}\left(u_{1} r-u_{2} r\right.}{\mathrm{d} r}\right]}{\mathrm{d} r}
\end{aligned}
$$

The velocities $u_{1}$ and $u_{2}$ follow from eliminating $v$ from eqs (1) and (2), integrating the result with respect to $r$, and applying the boundary conditions (8) and (9):

$$
u_{1}+u_{2}=\frac{\phi}{2 \pi r h} .
$$

Equation (15) is employed to eliminate $u_{1}$ from eq. (14):

$$
\begin{aligned}
& -\frac{m_{\mathrm{app}, \theta}}{k_{\theta}}\left[\left(\frac{\phi}{2 \pi h r}-u_{2}\right)^{n}-u_{2}^{n}\right] \\
& =\frac{\mathrm{d} \ln \left[\frac{1}{r} \frac{\mathrm{d}\left(\frac{\phi}{2 \pi h}-2 u_{2} r\right)}{\mathrm{d} r}\right]}{\mathrm{d} r} \\
& \times \frac{t_{f} m_{\mathrm{app}, ~} n}{k_{f}}\left[\frac{h}{4 r} \frac{\mathrm{d}\left(2 u_{2} r-\frac{\phi}{2 \pi h}\right)}{\mathrm{d} r}\right] .
\end{aligned}
$$

Equation (16) is rendered dimensionless by introduc-

$$
R=\frac{r}{r_{u}}
$$

and

$$
U_{2}=\frac{u_{2} 2 \pi r_{i} h}{\phi} .
$$

It can be verified that both dimensionless properties have values that lie between zero and unity. Substituting eqs (17) and (18) into eq. (16) produces

$$
-(a-y)^{n}+y^{n}=-A_{n}\left(\frac{\mathrm{d} y}{\mathrm{~d} R}\right)^{n-1}\left(\frac{1}{R} \frac{\mathrm{d} y}{\mathrm{~d} R}-\frac{\mathrm{d}^{2} y}{\mathrm{~d} R^{2}}\right)
$$

where

$$
y(R)=R U_{2}(R) .
$$

In eq. (19) the following dimensionless numbers have been introduced:

$$
\begin{gathered}
a=\frac{r_{i}}{r_{u}} \\
A_{n}=\frac{t_{f} m_{\mathrm{app}, f} n k_{g}}{r_{u} m_{\mathrm{app}, q} k_{f}}\left(\frac{h}{2 r_{u}}\right)^{n} .
\end{gathered}
$$

$A_{n}$ represents the filter material/filter gap flow resistance ratio and $a$ the inner-outer diameter ratio. The boundary conditions (8) and (9) pertaining to eq. (19) are transformed by combining cqs (8) and (9) with eqs (15), (18), (20) and (21):

$$
\begin{aligned}
& y(R=a)=0 \\
& y(R=1)=a .
\end{aligned}
$$

The solution of the boundary value problem described by eqs (19), (23) and (24) is obtained with a standard shooting method, which can be found e.g. in Hall and Watt (1976). For a Newtonian fluid (i.e. $n=1$ ), however, the equations can be solved in closed form, as will be discussed in the following.

For $n=1$ Kamke (1983) suggests the following substitutions:

$$
\begin{gathered}
y=R z(R)+\frac{1}{2} a \\
R=\left(\frac{A_{n}}{2}\right)^{1 / 2} x
\end{gathered}
$$

to transform eqs (19), (23) and (24) into

$$
\begin{gathered}
x^{2} \frac{\mathrm{d}^{2} z}{\mathrm{~d} x^{2}}+x \frac{\mathrm{d} z}{\mathrm{~d} x}-\left(1+x^{2}\right) z=0 \\
z\left[x=\left(\frac{2}{A_{n}}\right)^{1 / 2} a\right]=-\frac{1}{2} \\
z\left[x=\left(\frac{2}{A_{n}}\right)^{1 / 2}\right]=\frac{1}{2} a
\end{gathered}
$$

respectively. The solution of eq. (27) follows from Abramowitz and Stegun (1972) as

$$
z(x)=C_{1} I_{1}(x)+C_{2} K_{1}(x)
$$

where $I_{1}$ and $K_{1}$ are modified Bessel functions of the 
first order. The integration constants $C_{1}$ and $C_{2}$ fol-

low from boundary conditions (28) and (29):

$$
\begin{aligned}
& C_{1}=\frac{\frac{1}{2} K_{1}\left[\left(\frac{2}{A_{n}}\right)^{1 / 2}\right]+\frac{1}{2} a K_{1}\left[\left(\frac{2}{A_{n}}\right)^{1 / 2} a\right]}{I_{1}\left[\left(\frac{2}{A_{n}}\right)^{1 / 2}\right] K_{1}\left[\left(\frac{2}{A_{n}}\right)^{1 / 2} a\right]-K_{1}\left[\left(\frac{2}{A_{n}}\right)^{1 / 2}\right] I_{1}\left[\left(\frac{2}{A_{n}}\right)^{1 / 2} a\right]} \\
& C_{2}=\frac{-\frac{1}{2} I_{1}\left[\left(\frac{2}{A_{n}}\right)^{1 / 2}\right]-\frac{1}{2} a I_{1}\left[\left(\frac{2}{A_{n}}\right)^{1 / 2} a\right]}{I_{1}\left[\left(\frac{2}{A_{n}}\right)^{1 / 2}\right] K_{1}\left[\left(\frac{2}{A_{n}}\right)^{1 / 2} a\right]-K_{1}\left[\left(\frac{2}{A_{n}}\right)^{1 / 2}\right] I_{1}\left[\left(\frac{2}{A_{n}}\right)^{1 / 2} a\right]}
\end{aligned}
$$

Via different transformations Booy (1982) also obtained eqs (27)-(32).

A numerical solution method thus provides $y(R)$ for $n \neq 1$, and an analytical solution has been obtained for $n=1$. One of the most important physical properties to be determined is the total initial pressure drop in the filter, constituted by $p_{i}-p_{k}$.

This pressure drop will be minimal when the pressure drops in supply and discharge gaps tend to zero. In such an optimum case the liquid spreads uniformly over the medium and, hence, the minimum pressure drop reads

$$
\left(p_{i}-p_{u}\right)_{\min }=\frac{m_{\mathrm{app}, s} t_{f}}{k_{f}}\left[\frac{\phi}{2 \pi\left(r_{u}^{2}-r_{i}^{2}\right)}\right]^{\prime \prime}
$$

[see eq. (3)]. For a badly designed filter the actual pressure drop will be much larger as there is also a substantial pressure drop in the gaps, which docs not add to the filtration process.

The actual pressure drop is written as

$$
\begin{aligned}
p_{i}-p_{u} \equiv & p_{1}\left(r=r_{i}\right)-p_{2}\left(r=r_{u}\right) \\
= & p_{1}\left(r=r_{i}\right)-p_{2}\left(r=r_{i}\right) \\
& -\left(p_{2}\left(r=r_{u}\right)-p_{2}\left(r=r_{i}\right)\right)
\end{aligned}
$$

[see eqs (10) and (11)]. With eqs (13), (15), (17), (18), (20)-(22) and (33) the first term on the right-hand side is written as

$$
\frac{p_{l}-p_{2}\left(r=r_{i}\right)}{\left(p_{i}-p_{u}\right)_{\min }}=\left(\frac{1-a^{2}}{2 a}\right)^{n}\left[\left.\frac{1}{a} \frac{\mathrm{d} y}{\mathrm{~d} R}\right|_{R=a}\right]^{n} .
$$

With eqs (17), (18), (20)-(22) and (33) the second term on the right-hand side of eq. (34) is transformed into

$$
\frac{p_{2}\left(r=r_{i}\right)-p_{u}}{\left(p_{i}-p_{u}\right)_{\min }}=-\frac{n}{A_{n}}\left(\frac{1-a^{2}}{2 a}\right)^{n} \int_{a}^{1}\left(\frac{y}{R}\right)^{n} \mathrm{~d} R \text {. }
$$

The reciprocal of the dimensionless pressure drop $\Delta P$ is now obtained by adding eqs (35) and (36).

It is interesting to realize that the same differential equations are obtained if the fluid flows in the opposite direction, thus from the outer ring to the inner ring. This is in fact the (Newtonian) flow situation analyzed by Booy (1982). Equations (1), (2), (8) and (9) remain namely in the same form, while the right-hand sides of eqs (3)-(5) have to be multiplied by minus unity. Applying these equations, it is evident that the boundary problem (19), (23) and (24) remains the same as is the pressure drop.

\section{COMPUTATIONAL RESULTS}

In preceding sections the governing equations have been derived and rendered dimensionless. For a Newtonian liquid a solution in closed form has been derived, while for $n \neq 1$ the solution was obtained numerically. In this section computational results are presented for a broad range of the three governing dimensionless numbers of the process.

In Fig. 4 the resulting dimensionless pressure drop, $\Delta P$, is depicted for $A_{n}$ ranging from zero up to 2 , while $n=1$ and $a$ is set equal to 0.35 and 0.55 . In Fig. 5 the results pertaining to $n=0.55$ and all other unchanged values are plotted. These ranges of $A_{n}, a$ and $n$ extend well beyond a large number of practical applications.

Figures 4 and 5 indicate that for all situations the dimensionless pressure drop increases (i.e. the actual pressure drop decreases) with increasing $A_{n}$. This would be expected: larger $A_{n}$ implies that the flow resistance of the filter medium is much larger than the resistance of the gaps. Indeed, one can observe that for large $A_{n}$ the dimensionless pressure drop approximates unity, i.e. the actual pressure drop approximates the minimum pressure drop. In this case indeed the velocity $v(r)$ hardly depends on the radial coordinate, that is to say, the fluid uniformly spreads over the filter medium. For small $A_{m}$, however, the pressure drop across the filter is enhanced by a substantial resistance of the gaps, which do not add to the filtration process. Hence, stacked-disc filters with such small $A_{n}$ can be considered to be badly designed.

For large inner-outer diameter ratios the enhancement of $\Delta P$ is more pronounced for small $A_{n}$. Large diameter ratios implies a large $r_{i}$ and, hence, a smaller velocity and related pressure drop near the entrance. For small inner-outer diameter ratios, on the other hand, the diverging flow effect is large and, therefore, $\Delta P$ is less sensitive to augmenting $A_{n}$.

Figures 4 and 5 reveal furthermore that for identical $A_{n}$ and $a$, the power-law fluid $(n<1)$ seems to bring about a smaller pressure drop ( $\Delta P$ is larger). However, one must realize that for smaller $n$ the value of $A_{n}$ also diminishes [see eq. (22)]. Figures 4 and 5 indicate that 


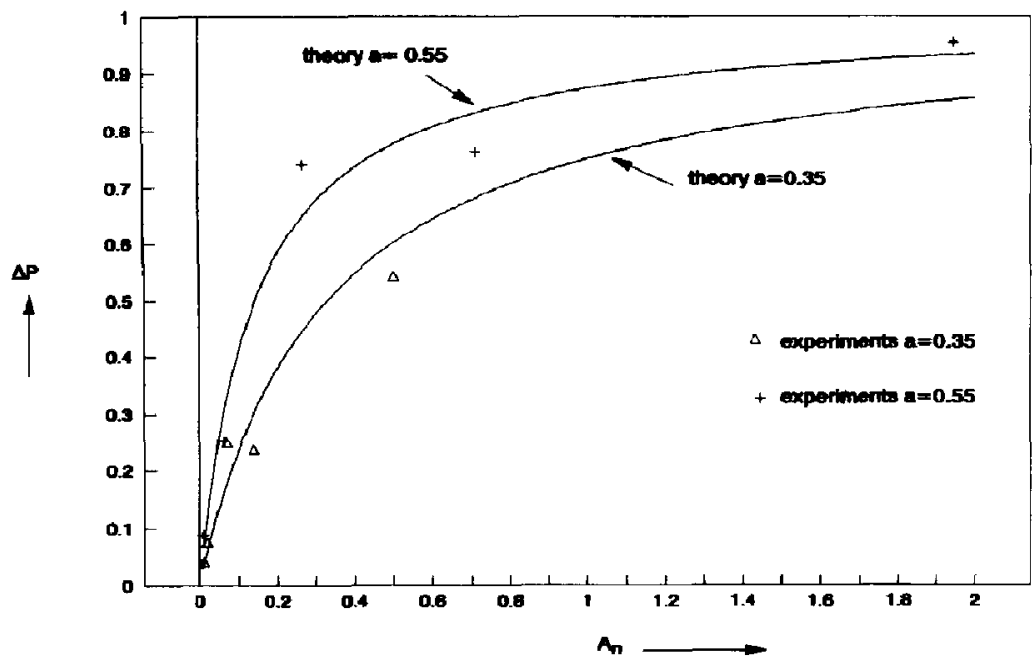

Fig. 4. Dimensionless pressure drop for various $A_{n}, a=0.35$ and 0.55 , and $n=1$, according to the theoretical model and experiments.

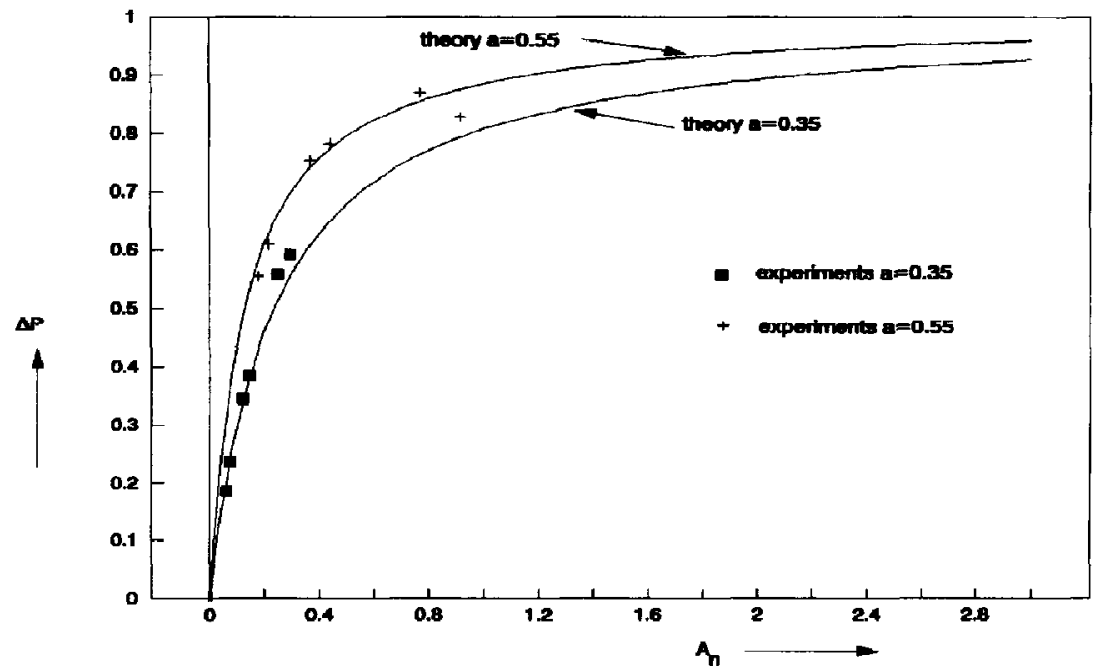

Fig. 5. Dimensionless pressure drop for various $A_{n}, a=0.35$ and 0.55 , and $n=0.55$, according to the theoretical model and experiments.

small $A_{n}$ imply large pressure drops. So, it is difficult to assess the actual effect of shear thinning behavior on pressure drop across the filter.

The results of the present analysis can also be fruitfully used for the scale-up of stacked-disc filters. Consider a fluid flowing through a filter with certain $A_{n}$, diameter ratio $a$ and power-law index $n$ values, and a corresponding pressure drop. This filter is scaled-up with the requirement that the scaled-up version contains the same dimensionless pressure drop. This will be the case if $A_{n}$, the diameter ratio $a$ and the power-law index $n$ remain unchanged. As the same fluid is filtered, $n$ remains the same. Usually, the inner-outer diameter ratio $a$ is varied modestly or even not varied at all, so that this group is a constant too. Hence, $A_{n}$ is the only dimensionless group which is affected by scale-up [see eq. (22)].

Since the filter medium is prescribed by the filtration efficiency, the medium (and its corresponding values of $k_{f}$ and $t_{f}$ ) remains the same. Now it follows that for the scaled-up filter

$$
\underset{\text { scale-up }}{\frac{m_{\text {app }, f} n k_{g}}{r_{u} m_{\text {app.e }}}}\left(\frac{h}{2 r_{u}}\right)^{n}=\frac{m_{\text {spp }, f} n k_{g}}{r_{u} m_{\text {app }, g}}\left(\frac{h}{2 r_{u}}\right)^{n}
$$

In the case of gaps filled with support material, $k_{\theta}$ does 
not vary with $h$; eq. (37) therefore reduces to

$$
\begin{gathered}
\frac{m_{\text {app }, f} n}{r_{u} m_{\text {app } . g}}\left(\frac{h}{2 r_{u}}\right)^{n}= \\
\text { scale-up }
\end{gathered}
$$

For open gaps, on the other hand, $k_{g}$ and $m_{\mathrm{app} . g}$ are directly related to $h$ and $n$ by eqs (6) and (7). For these filters eq. (37) becomes

$$
\begin{aligned}
& \frac{m_{\mathrm{app} . f n h}}{m r_{u}}\left(\frac{h^{2} n}{2(2+4 n) r_{u}}\right)^{n}=\frac{m_{\mathrm{app} .} n h}{m r_{u}}\left(\frac{h^{2} n}{2(2+4 n) r_{u}}\right)^{n} \\
& \text { scale-up } \\
& \text { model }
\end{aligned}
$$

For Newtonian fluids both apparent viscosities and $m$ will drop out of eqs (37)-(39). Equations (38) and (39) are compact and useful results for a proper scaleup of stacked-disc filters.

\section{EXPERIMENTS}

In this section the theoretical clean pressure drop predictions are compared with the results of performed experiments. To determine the pressure drop for various values of the dimensionless groups, $a, A_{n}$ and $n$, different filter materials, support screens and model liquids have been employed. The used liquids, polybutylene and a solution of acrylic acid polymer in water, are listed in Table 1, together with their
Table 1. Properties of the model liquids $\left(20^{\circ} \mathrm{C}\right)$ used for experiments (*Keyzer and Mackay, ${ }^{* *}$ B. F. Goodrich)

\begin{tabular}{lcl}
\hline Composition & $m\left(\mathrm{~Pa} \mathrm{~s}^{n}\right)$ & $n$ \\
\hline Indopol $\mathrm{H} \mathrm{100^{* }}$ & 30 & 1 \\
Carbopol $941^{* *}(0.25$ mass \% $)$ in water & 0.27 & 0.55 \\
\hline
\end{tabular}

rheological properties, which were measured with a Rheomat $135 \mathrm{~S}$.

With the aid of polybutylene, Indopol H100, and flow-pressure drop measurements, the permeability of the filter media were determined with a device depicted in Fig. 6. The pressure drop was measured with a Druck pressure gauge and the flow with a weighing machine and a chronometer. The permeability simply follows from eq. (33), where $m_{\text {app, } f}=m$ and $n=1$. The permeability and porosity of the filter layers involved are listed in Table 2.

The permeability of the support screens was obtained with the help of the device depicted in Fig. 7 and polybutylene. The permeability follows from

$$
k_{g}=\frac{m \phi \ln \left(\frac{r_{u}}{r_{i}}\right)}{2 \pi\left(p_{i}-p_{u}\right) h} .
$$

This equation can be derived from eq. (4) [or eq. (5)],

Iable 2. Permeability, porosity and apparent viscosity $(n=0.55)$ of the used filter layers (*Bekaert, **MGW Dinxperlo).

\begin{tabular}{lclc}
\hline Filter material & $k_{f}\left(\mathrm{~m}^{2}\right)$ & $\varepsilon$ & $\frac{m_{\text {app }, f}}{m}\left(\mathrm{~m}^{1-n}\right)$ \\
\hline Bekipor 10AL3AA* & $1.24 \times 10^{-11}$ & 0.75 & \\
Bekipor 25AL2AA* & $5.99 \times 10^{-11}$ & 0.72 & \\
Bekipor ST25AL2* & $2.49 \times 10^{-11}$ & 0.72 & \\
Mesh 325** & $5.56 \times 10^{-11}$ & 0.31 & \\
Köper Tressen 15** & $0.54 \times 10^{-11}$ & 0.13 & $6.07 \times 10^{-2}$ \\
Köper Tressen 10** & $0.27 \times 10^{-11}$ & 0.07 & $4.36 \times 10^{-2}$ \\
Köper Tressen 5** & $0.67 \times 10^{-12}$ & 0.11 & $4.29 \times 10^{-2}$ \\
\hline
\end{tabular}

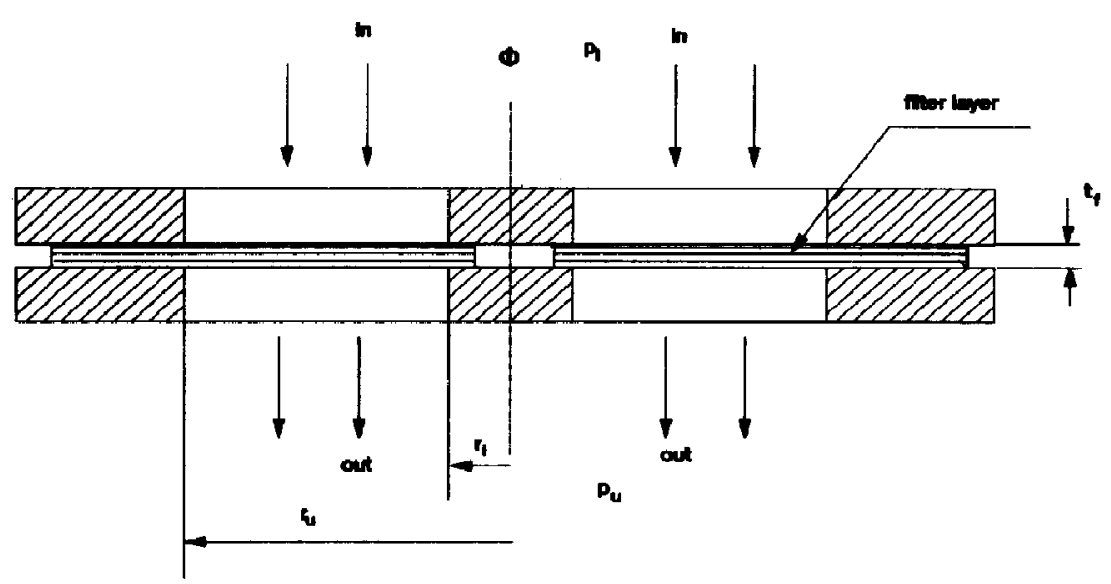

Fig. 6. Device for measuring both the permeability of the filter layer and the apparent viscosity of non-Newtonian fluid flow across the layer. 
eq. (1) [or eq. (2)], eq. (8) [or eq. (9)], eqs (10) and (11), $v=0, n=1$ and $m_{\text {app., }}=m$. In Table 3 the permeability and porosity of the employed support screens are summarized.

The support screens and filter layers were assembled to form one layer of a stacked-disc filter with variuus $A_{n}$ and $a=0.35$ and $a=0.55$ (see Fig. 8).

Table 3. Permeability, porosity and apparent viscosity $(n=0.55)$ of the used support screens (*MGW Dinxperlo)

\begin{tabular}{lccc}
\hline Support screen & $k_{8}\left(\mathrm{~m}^{2}\right)$ & $\varepsilon$ & $\frac{m_{\text {app } * \theta}}{m}\left(\mathrm{~m}^{1-n}\right)$ \\
\hline Mesh $5^{*}$ & $8.37 \times 10^{-8}$ & 0.39 & \\
Mesh 10* & $8.27 \times 10^{-8}$ & 0.23 & \\
Mesh 60* & $1.00 \times 10^{-9}$ & 0.17 & $8.79 \times 10^{-4}$ \\
Mesh 120* & $0.17 \times 10^{-9}$ & 0.11 & $2.91 \times 10^{-4}$ \\
\hline
\end{tabular}

Figure 4 provides the experimental dimensionless pressure drops, obtained with the polybutylene measurements. One can readily see the close agreement between the theoretical model and the experimental results, both for small $A_{m}$ (high pressure drops) and for larger $\boldsymbol{A}_{n}$ (smaller pressure drops, implying a better filter layout).

A complication with power-law liquids is the appearance of $m_{\mathrm{app}, \mathrm{g}}$ and $m_{\mathrm{app}, f}$ in $A_{n}$ [see eq. (22)]. The apparent viscosity of the used solution of acrylic acid polymer (Carbopol 941) in water flowing through

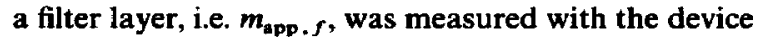
depicted in Fig. 6 and with the help of eq. (33). The obtained $m_{\text {app,g }} / m$ are tabulated in Table 2. As expected, the measured values of $p_{i}-p_{u}$ and $\phi^{n}$ $(n=0.55)$ indeed lie on a straight line.

The appparent viscosity $m_{\mathrm{app}, g}$ was determined with the aid of the pressure drop, and the flow

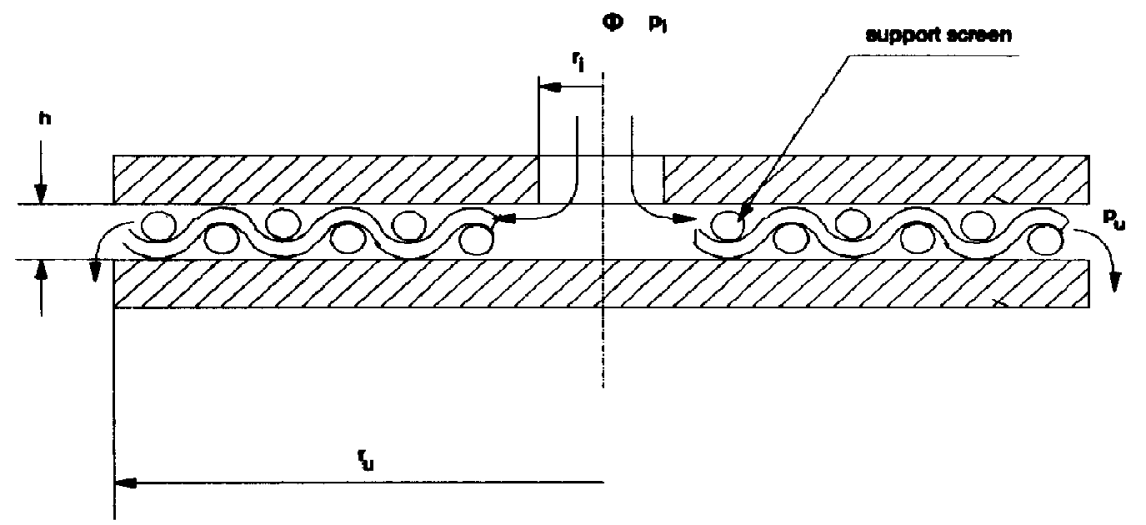

Fig. 7. Device for measuring both the permeability of the support screen and the apparent viscosity of non-Newtonian fluid flow across the screen.

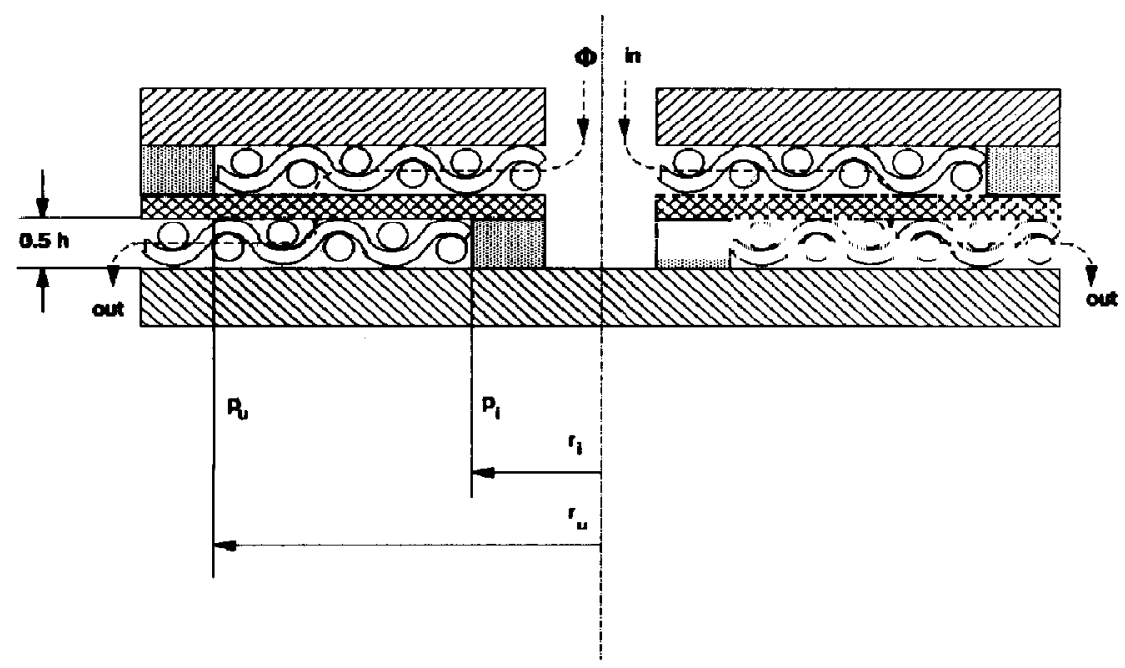

Fig. 8. Disc filter tested. 
measurements using the device of Fig. 7 and the following equation:

$$
m_{\text {app .q }}=\left(\frac{2 \pi h}{\phi}\right)^{n} \frac{(1-n) k_{g}\left(p_{i}-p_{u}\right)}{\left(r_{u}^{1-n}-r_{i}^{1-n}\right)} .
$$

This equation can be derived from eq. (4) [or eq. (5)], eq. (1) [or eq. (2)], eq. (8) [or eq. (9)], eqs (10) and (11) and $v=0$. Various flow and pressure drop measurements for each support screen yield $m_{\mathrm{app} . p}$, which are given in lable 3. Again $p_{i}-p_{u}$ and $\phi^{n}$ were linearly dependent. It is worthwhile to remark that indeed $m_{\text {app.e }}$ of eq. (41) tends asymptotically to $m$ of eq. (40) if $n$ approximates unity. This is elucidated by considering

$$
\begin{aligned}
\operatorname{Lim}_{n \rightarrow 1} \frac{1-n}{r_{u}^{1-n}-r_{i}^{1-n}} & =\operatorname{Lim}_{n \rightarrow 1} \frac{-1}{-r_{u}^{1-n} \ln r_{u}+r_{i}^{1-n} \ln r_{i}} \\
& =\left[\ln \left(\frac{r_{u}}{r_{i}}\right)\right]^{-1}
\end{aligned}
$$

where l'Hôpitals rule has been applied.

For various $A_{n}$ and ratios $a$ the experimental flow and pressure drop across the disc layer are determined. In some cases the ratio $m_{\text {app }, f} / m_{\text {app }, \theta}$, figuring in $A_{n}$, amounted to a value of $10^{2}$, which illustrates the true non-Newtonian character of the experiments [as said, in the Newtonian case $m_{\mathrm{app} . r} / m_{\mathrm{app.q}}=1$ and then this ratio drops out of eq. (22)]. The experimental dimensionless pressure drops are shown in Fig. 5, once again illustrating the precision of the theoretical model.

\section{CONCLUSIONS}

In this paper the clean non-Newtonian flow and the related pressure drop across stacked-disc filters have been investigated both theoretically and experimentally. The flow was described by Darcy's law and the non-Newtonian liquid was regarded as a powerlaw fluid. The governing equation was rendered dimensionless and then solved. The solution is governed by three dimensionless groups, viz. the power-law index $n$, the inner-outer diameter ratio $a$, and filter material/filter gap flow resistance ratio $A_{n}$.

The computational results revealed the increased pressure loss across the filter for small $A_{n}$ and $a$. These results give an insight into the optimum construction of stacked-disc filters. On the basis of the present analysis compact rules are proposed for the proper scale-up of these filters.

For $n=1$ and $n=0.55, a=0.35$ and $a=0.5$, and $0.01 \leqslant A_{n} \leqslant 0.92$ the theoretical predictions have been compared with the obtained experimental results. To this end, one-layer stacked-disc filters were constructed of various filter layers and support screens. Through these constructed filters, both acrylic acid polymer dissolved in water and polybutylene were forced to flow. The outcome of the theoretical model of the filter appears to be in close agreement with the experimental results.

Acknowledgement - The authors thank Mr. G. Vegt for his support of this work and the management of Akzo Research

Laboratories Arnhem for their permission to publish this paper. They also thank Messrs $P$. Den Decker and $H$. Knoester, who provided mathematical advice. The experimental assistance of Messrs. P. J. Van Leijen, E. Heuveling, F. M. A. Van Der Mast and R. E. Burgers is greatly acknowledged.

$A_{n}$
$C_{1}, C_{2}$
$h$
$I_{1}, K_{1}$
$k$
$m_{\mathrm{app}}$
$m$
$n$
$\left(p_{i}-p_{u}\right)_{\min }$

$\boldsymbol{P}$

$\boldsymbol{R}$

$r$

$t_{f}$

$U_{2}$

$\boldsymbol{u}$

$v$

$x$

$\boldsymbol{y}$

$\boldsymbol{z}$

Greek letter
$\Delta P$
$E$
$\phi$
Subscripts
$f$
$g$
$i$
$u$
1
2

Greek letters

$\Delta P \quad$ dimensionless pressure drop,

$\left(p_{i}-p_{u}\right)_{\min } /\left(p_{i}-p_{u}\right)$

porosity

fluid volume flow per disc

filter medium

supply and discharge gap

inner ring

outer ring

supply gap

discharge gap

\section{REFERENCES}

Abramowitz, M. and Stegun, I. A., 1972, Handbook of Mathematical Functions. Dover, New York.

Berman, A. S., 1953, Laminar flow in channels with porous walls. J. Appl. Phys. 24, 1232-1235.

Bird, R. B., Armstrong, R. C. and Hassager, O., 1977, $D y-$ namics of Polymeric Liquids, Vol. 1: Fluid Mechanics. J. Wiley, New York.

Booy, M. L., 1982, Flow, pressure distribution, and internal forces in stacked-disc filter. Polym. Engng Sci. 22, 895-901.

Darcy, H. P. G., 1856, Les Fontaines Publiques de la Ville de Dijon. Victor Dalmont, Paris (in French).

Elghossain. M. J., 1990. Residence time distribution in a melt polymer filter. Paper presented at the American Filtration Society Washington Conference. 
Hall, G. and Watt, J. M., 1976, Modern Numerical Methods for Ordinary Differential Equations. Clarendon Press, Oxford.

Kamke, E., 1983, Differentialgleichungen, Lösungsmethoden und Lösungen (10. Aufl.). Teubner, Stuttgart (in German).

Kaplan, S. J., Morland, C. D. and Hsu, S. C., 1979. Predict
non-Newtonian fluid pressure drop across random-fibre filters, Chem. Engng, 27 August, 93-98.

Kozicki, W. and Tiu, C., 1988, A unified model for nonNewtonian flow in packed beds and porous media, Rheolog. Acta 27, 31-38. 